\title{
The Effect of Positioning Exercises on Dyspnea and Fatigue in Patients Treated with COVID-19
}

\author{
Jaspreet Kaur Kang ${ }^{1}$, Neeta J Vyas ${ }^{2}$ \\ ${ }^{1} \mathrm{PhD}$ Scholar, Gujarat University and Principal, Mahatma Gandhi Physiotherapy College, Memco- Naroda \\ Road, Ahmedabad \\ ${ }^{2}$ Professor, Ahmedabad Institute of Medical Sciences, Lapkaman, Ahmedabad \\ Corresponding Author: Jaspreet Kaur Kang
}

\begin{abstract}
Introduction: Coronavirus disease 2019 (COVID-19) - a global pandemic has severely affected humankind. It basically affects both the upper (i.e. sinuses, nose and throat) and lower (i.e. trachea and lungs) airways hence causing respiratory tract diseases ranging from asymptomatic or cold to more severe lung diseases .Since it affects the respiratory system, so Dyspnea and fatigue are one of the most prominent symptoms for COVID-19. The aim of the study is to determine the effect of positioning exercises in COVID-19 patients which can contribute to relieve dyspnea and reduce fatigue

Methodology: 80 Patients, age range 18 to 50 years, diagnosed with Covid 19 and undergoing treatment for the same with grade $\leq 3$ NYHA dyspnea classifications were included and divided equally in two groups-Groups I (experimental) and Group II (Control). In Group I positioning exercises were administered 30 minutes twice a day for 7 days along with the regular Covid treatment protocol and in Group II followed regular Covid treatment protocol. Pre and post evaluation of oxygen saturation and respiratory rate with fatigue severity scale were taken

Results: As the data was not normally distributed non parametric test was used. Wilcoxon test was used to compare the outcome measures within groups and Man-Whitney test was used for comparing between groups. Result shows that there was significant improvement noted in both the groups within and between comparison but Group I was found to be highly significant then Group II.
\end{abstract}

Conclusion: Positioning exercises in COVID-19 patients helps to relieve dyspnea and reduce fatigue.

Key Words: positioning, oxygen saturation, respiratory rate, fatigue impact scale, covid-19 patients.

\section{INTRODUCTION}

In Wuhan, China a novel human Coronavirus (SARS-CoV-2) was identified in late 2019, which was an outbreak of respiratory disease. ${ }^{1}$ Corona virus spread to a large extent via respiratory droplets that belongs to the $\beta$-corona cluster. ${ }^{2}$ People infected with Covid 19 with mild to moderate disease was found to be approximately $80 \%$ and severe disease that can lead to hypoxic respiratory failure and acute respiratory distress syndrome (ARDS) was found to be more then $10 \%{ }^{3}$ Severe patients can require invasive mechanical ventilation (IMV) and hospitalization for a long period caused by a viral pneumonia with clinical features that parallel the acute respiratory distress syndrome (ARDS). Conventional ARDS, characterized by diffuse alveolar infiltrates, noncompliant lungs, and increased dead space ventilation, is associated with profound hypoxemia, significant morbidity, and substantial mortality. ${ }^{4}$

It ranges from asymptomatic or cold to more severe lung diseases as it basically affects both the upper (i.e. sinuses, nose and throat) and lower (i.e. trachea and lungs) airways hence causing respiratory tract diseases. Since it affects the respiratory 
system, so Dyspnea and fatigue are one of the most prominent symptoms for COVID19. ${ }^{4}$ Many COVID-19-infected patients have profound desaturation (blood oxygen saturations $<80 \%$ ) but only modest symptoms, the term "happy hypoxemics" has evolved to describe individuals with this disconnect between their physiology and symptoms. ${ }^{5}$

The Fatigue Impact Scale (FIS) was developed to assess the symptom of fatigue as part of an underlying chronic disease or condition. Consisting of 40 items, each of which is scored from 0 (no problem) to 4 (extreme problem), providing a continuous scale of $0-160$. It evaluates the effect of fatigue on three domains of daily life: cognitive functioning, physical functioning, and psychosocial functioning. ${ }^{6}$

Chest physiotherapy provides airway clearance techniques, optimal patient positioning, and inspiratory muscle training that improve the ventilatory function in these patients. Hence the aim of the present study is to determine effect of positioning exercises on dyspnea and Fatigue Impact scale in patient treated with Covid 19.

\section{MATERIALS AND METHODOLOGY}

An experimental study was done on 50 patients with the age group between 18 to 50 years. Inclusion criteria were as follows: laboratory-confirmed COVID-19, patients undergoing treatment for the same with grade $\leq 3$ NYHA dyspnea. Patient unwilling to participate, patients having unstable vitals, patients with altered mental status and with inability to turn in bed without assistance,with extreme respiratory distress requiring immediate intubation were excluded from the study. Materials used in the study were data collection sheet, consent form, pulse oximeter and Timer.

Patients were randomly selected; detail explanation regarding study was given to patients and their relatives. Consent was taken by the patient and their relative. An Ethical Approval was obtained from the respective hospital for conducting the study. Total 50 patients were included in the study,
25 in each group and were further divided into two groups.

In Group I (Experimental Group) Positioning exercises were administered for 30 minutes twice a day for 7 days along with regular Covid treatment . The positions used were as follows: proning, side lying (both sides) and semi fowler's position. Group II (Control Group) Regular Covid treatment Protocol was given for 7 days. The regular protocol included medications, breathing exercises, coughing and suctioning. Before and after Treatment Protocol, all Outcomes measures including Oxygen Saturation, Respiratory Rate and Fatigue Impact Scale were taken.

Statistical analysis: SPSS version 2.0 was used for analysis. As the data was not normally distributed non parametric test was used. Wilcoxon test was used to compare the outcome measures within groups and Man-Whitney was used for comparing between groups. Significance level was fixed at $p<0.05$.

\section{RESULTS}

Table 1: Effect of positioning exercise on SPO2, RR, and FIS in GROUP I

\begin{tabular}{|l|l|l|l|l|l|}
\hline Group & Variable & $\begin{array}{l}\text { Pre } \\
\text { mean }\end{array}$ & $\begin{array}{l}\text { Post } \\
\text { mean }\end{array}$ & $\begin{array}{l}\text { p } \\
\text { value }\end{array}$ & Significance \\
\hline & SPO2 & 87.03 & 98.73 & 0.001 & significant \\
\hline I & RR & 25.03 & 14.8 & 0.003 & significant \\
\hline & FIS & 41.7 & 27.4 & 0.002 & significant \\
\hline
\end{tabular}

Table 2: Effect of positioning exercise on SPO2, RR, and FIS in Group II

\begin{tabular}{|l|l|l|l|l|l|}
\hline Group & Variable & $\begin{array}{l}\text { Pre } \\
\text { Mean }\end{array}$ & $\begin{array}{l}\text { Post } \\
\text { Mean }\end{array}$ & $\begin{array}{l}\text { p- } \\
\text { Value }\end{array}$ & Significance \\
\hline & SpO2 & 86.79 & 93.07 & 0.005 & Significant \\
\hline II & RR & 28.15 & 18.32 & 0.004 & Significant \\
\hline & FIS & 42.9 & 32.25 & 0.003 & Significant \\
\hline
\end{tabular}

\section{Mean}

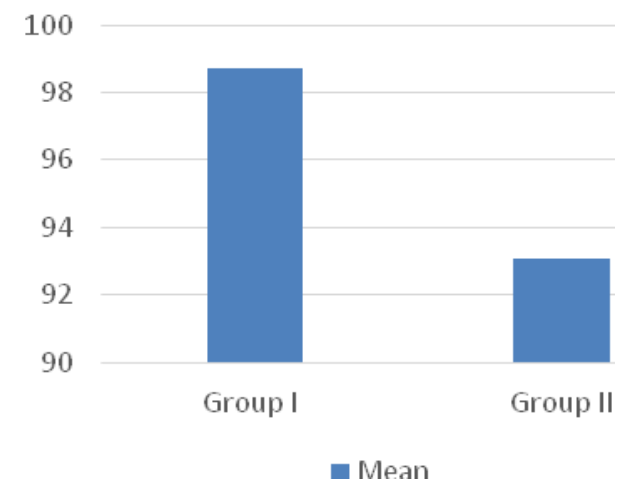

Graph 1: Shows between groups Comparison 
There was significant improvement noted on Oxygen Saturation, Respiratory Rate and Fatigue Impact Scale within groups. Moreover, significant result were noted while comparing between the group but Group I was found to be significant then Group II.

\section{DISCUSSION}

The current study was conducted to determine the effect of positioning exercises on dyspnea and fatigue impact scale in patients treated with Covid 19. Results showed that there was significant improvement noted within group and also while comparing between group, but Group I shows more significance then Group II as body positioning increases regional ventilation-perfusion, improves alveolar ventilation, decrease airway resistance, increase lung compliance, decrease work of breathing, decrease preload and after load and decrease the work of heart. Hence there was significant improvement noted on oxygen saturation, respiratory rate and fatigue impact scale after 7 days of treatment protocol. ${ }^{7}$

The present study was further supported by Mahendra Damarla et al, in 2020 and concluded that there was improvement in oxygen saturation in nonintubated patients by prone positioning together with a combined strategy of High flow Nasal Cannula (HFNC) and restrictive fluid or non-invasive ventilation. ${ }^{8}$ Also study done on $7^{\text {th }}$ July 2020 by Giuseppe Fotii that prone positioning may improve oxygenation in patient with Covid -19 related pneumonia and concluded that oxygen saturation was significantly improved by prone positioning in Covid-19 parients. ${ }^{9}$

Barkar J et al (2020) concluded that, in awake, spontaneously breathing patients with COVID-19 severe hypoxemic respiratory failure, use of prone positioning was associated with improved oxygenation. In addition, there was lower rate of intubation in patients with an Spo2 of $95 \%$ or greater after 1 hour of the prone position. ${ }^{10}$ The present study also shows significant improvement on respiratory rate which was further supported by Sartini $C$ et al (2020) concluded that compared to baseline the respiratory rate was lower and the oxygenation was higher during and after pronation. ${ }^{11}$

Thus the present study concluded that Positioning exercises can be considered as an effective method for better improvement by relieving dyspnea and fatigue in COVID 19 treated in addition to the regular medical treatment.

Limitation of the study was that equal gender distribution was not considered and moreover patients on ventilator were not included. Comparison of different positions on oxygen saturation, $\mathrm{RR}, \mathrm{BP}$, pulse rate etc can also be done for future studies with a larger sample size in various respiratory conditions.

\section{CONCLUSION}

Positioning exercises can be considered as an effective method for improvement in relieving dyspnea and fatigue in COVID 19 treated patients in addition to the regular medical treatment.

\section{ACKNOWLEDGEMENT}

Special thanks to all the subjects who participated in the study to make this study successful. I want to thanks my parents and colleagues for motivation and their help throughout my study. I am thankful to my guide Dr. Neeta Vyas for all their guidance and support.

\section{Conflict of Interest: None}

\section{Source of Funding: None}

\section{Ethical Approval: Approved}

\section{REFERENCES}

1. Battaglini D, Robba C, Caiffa S, et al. Chest physiotherapy: An important adjuvant in critically ill mechanically ventilated patients with COVID-19. Respir Physiol Neurobiol. 2020;282:103529.

doi:10.1016/j.resp.2020.103529 
2. Auwal Abdullahi ${ }^{1,2 *}$ Safety and Efficacy of Chest Physiotherapy in Patients With COVID-19: A Critical Review. Front. Med., 21 July 2020 https://doi.org/10.3389/fmed.2020.00454.

3. Respiratory Physiotherapy in Severe COVID-19 Patients (FTR-COVID) July 7, 2020 U.S National liberary of Medicine.

4. Fabio Perrotta, Graziamaria Corbi, et al, (2020). Pulmonary Rehabilitation and Physiotherapy Management of Respiratory Conditions in Patient with COVID-19: Narrative Review. Military Caring sciences, 7(1), 63.10.29252/mcs.7.1.63.

5. Carfî A, Bernabei R, Landi F, for the Gemelli Against COVID-19 Post-Acute Care Study Group. Persistent Symptoms in Patients After Acute COVID-19. JAMA. 2020;324(6):603-605. doi:10.1001/jama.2020.12603.

6. Vanage, S. M., Gilbertson, K. K., \& Mathiowetz, V. (2003). Effects of an energy conservation course on fatigue impact for persons with progressive multiple sclerosis. The American Journal of Occupational Therapy, 57 (3), 315-323.

7. Elizabet Donna. Principles and practice of cardiopulmonary physical therapy. Third edition.
8. Mahendra Damarl, Shannon Niedermeyer et al, Prone Positioning of Nonintubated Patients with COVID-19.Americian Journal of respiratory and critical care medicine. 2020. Volume 202, Issue 4.

9. Guérin C, Reignier J, Richard J-C, et al. Prone positioning in severe acute respiratory distress syndrome. N Engl J Med 2013; 368: 2159-216

10. Barker J, Pan D, Koeckerling D, et al Effect of serial awake prone positioning on oxygenation in patients admitted to intensive care with COVID-19.Postgraduate Medical Journal Published Online First: 30 April 2021. doi: 10.1136/postgradmedj2020-139631

11. Sartini C, Tresoldi M, Scarpellini P, et al. Respiratory Parameters in Patients With COVID-19 After Using Noninvasive Ventilation in the Prone Position Outside the Intensive Care Unit. JAMA. 2020; 323(22):2338-2340.

doi:10.1001/jama.2020.7861

How to cite this article: Kang JK, Vyas NJ. The effect of positioning exercises on dyspnea and fatigue in patients treated with COVID-19. Int $J$ Health Sci Res. 2021; 11(10):1-4. DOI: https://doi.org/10.52403/ijhsr.20211001 